\title{
Constraints on the Jet-Medium Coupling from Measurements at RHIC and LHC
}

\author{
Barbara Betz $^{\mathrm{a}}$, Florian Senzel ${ }^{\mathrm{a}}$, Carsten Greiner ${ }^{\mathrm{a}}$, Miklos Gyulassy ${ }^{\mathrm{b}}$ \\ ${ }^{a}$ Institute for Theoretical Physics, Johann Wolfgang Goethe-University, 60438 Frankfurt am Main, Germany \\ ${ }^{b}$ Department of Physics, Columbia University, New York, 10027, USA
}

\begin{abstract}
The measured data on the nuclear modification factor for pions and reconstructed jets as well as on the high- $p_{T}$ elliptic flow at RHIC and LHC energies are compared to results from a linear pQCD and a highly non-linear hybrid AdS holographic model of jet-energy loss. We find that the high- $p_{T}$ ellitic flow requires to include realistic medium transverse flow fields and a jet-medium coupling including the effects of the energy of the jet, the temperature of the bulk medium, and non-equilibrium effects close to the phase transition. We extend our jet-energy loss model that is coupled to state-of-the-art hydrodynamic prescriptions to backgrounds generated by the parton cascade BAMPS. We demonstrate that the results for the hydrodynamic and the parton-cascade backgrounds show a remarkable similarity. Unfortunately, the results for both the pion and a parton-jet nuclear modification factor are insensitive to the jet-path dependence of the models considered.
\end{abstract}

Keywords: Jet Quenching, Viscous Hydrodynamics, Transport Model, Jet Holography

\section{Introduction}

One of the formidable tasks in heavy-ion physics is to identify a precise understanding of the jet-medium dynamics, the jet-medium interactions, and the jet-energy loss formalism. Below, we study the influence of the details of the jet-medium coupling and the medium background on the simultaneous description of the nuclear modification factor $\left(R_{A A}\right)$ and the high- $p_{T}$ elliptic flow $\left(v_{2}\right)$ measured at RHIC and LHC [1, 2, 3, 4] for a radiative pQCD energy-loss ansatz [5]. We contrast media determined via the viscous hydrodynamic approach VISH2+1 [6] with the parton-cascade BAMPS [7] as well as a jet-medium coupling depending on the collision energy with a jet-medium coupling influenced by the energy of the jet, the temperature of the medium and non-equilibrium effects around the phase transition.

Besides this, we compare the jet-energy loss based on radiative pQCD [5] with the Hybrid AdS energy-loss ansatz of Ref. [8]. We contrast the pion nuclear modification factor obtained via the radiative pQCD-energy loss [5] and the Hybrid AdS energy-loss ansatz with a parton-jet nuclear modification factor that can be con- sidered as an idealized LO Jet $R_{A A}$ at RHIC and LHC energies.

The pQCD-based energy loss model studied is parametrized as [5]

$$
\frac{d E}{d x}=\frac{d E}{d \tau}\left(\vec{x}_{0}, \phi, \tau\right)=-\kappa E^{a}(\tau) \tau^{z} e^{c=(2+z-a) / 4} \zeta_{q} v_{f},
$$

with the jet-energy dependence $a$, the path-length dependence $z$, and the energy dependence $c$. In the following, the jet-medium coupling $\kappa$ will depend either on the collision energy $\kappa=\kappa\left(\sqrt{s_{N N}}\right)$ or the energy of the jet and the temperature of the background medium considered $\kappa=\kappa\left(E^{2}, T\right)$. The jet-energy loss fluctuations are distributed via $f_{q}\left(\zeta_{q}\right)=\frac{(1+q)}{(q+2)^{1+q}}\left(q+2-\zeta_{q}\right)^{q}$, allowing for an easy interpolation between non-fluctuating $\left(\zeta_{q=-1}=1\right)$, uniform Dirac distributions and distributions increasingly skewed towards small $\zeta_{q>-1}<1$.

The jets are spread according to a transverse initial profile specified by the bulk flow fields given by the VISH2+1 and BAMPS backgrounds considered [6, 7].

On the other hand, the jet-energy loss of the Hybrid AdS energy-loss ansatz [8] is based on falling strings 

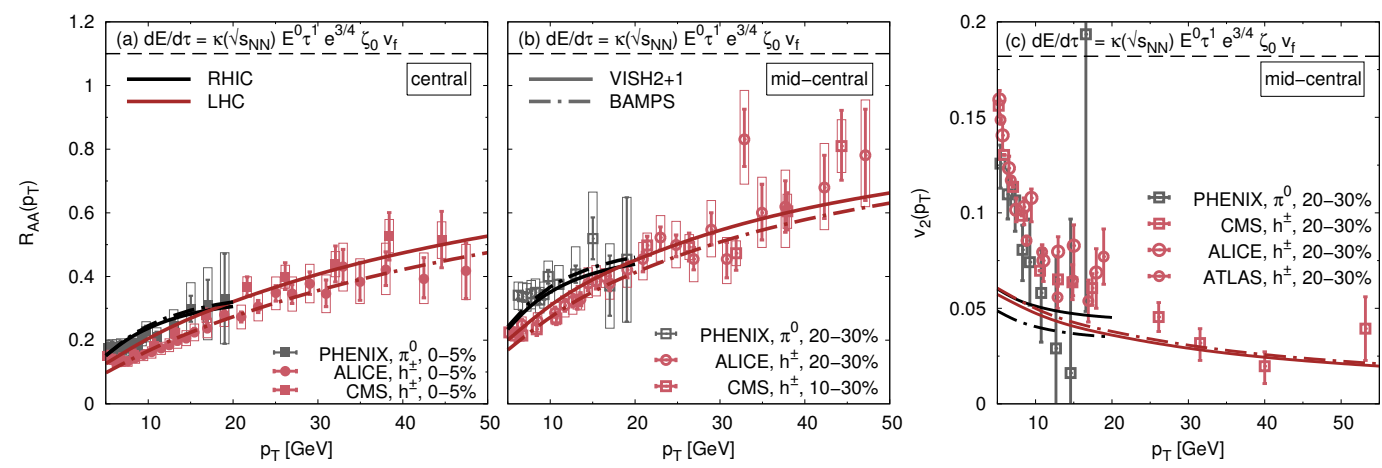

Figure 1: The pion nuclear modification factor for central (left panel) and mid-central (middle panel) collisions at RHIC (black) and LHC (red) as well as the high- $p_{T}$ pion elliptic flow for mid-central events (right panel). The measured data [1 2 | $3 \mid$ 4| is compared to a pQCD-based energy loss $d E / d \tau=\kappa\left(\sqrt{s_{N N}}\right) E^{0} \tau^{1} e^{3 / 4} \zeta_{0} v_{f}$, including jet-energy loss fluctuations $\left(\zeta_{0}\right)$ and transverse flow fields $\left(v_{f}\right)$, for a jet-medium coupling that depends on the collision energy $\left(\kappa=\kappa\left(\sqrt{s_{N N}}\right)\right)$ applying the hydrodynamic backgrounds of VISH2+1 [6] (solid lines) and the parton cascade BAMPS [7] (dashed-dotted lines).

[9] where

$$
\frac{1}{E_{\text {in }}} \frac{d E}{d x}=-\frac{4}{\pi} \frac{x^{2}}{x_{\text {stop }}^{2}} \frac{1}{\sqrt{x_{\text {stop }}^{2}-x^{2}}} .
$$

The initial jet energy is given by $E_{\text {in }}$ and the string stopping distance for quark and gluon jets is determined via $x_{\text {stop }}^{q, g}=1 /\left(2 \kappa_{\mathrm{sc}}^{q, g}\right) E_{\mathrm{in}}^{1 / 3} / T^{4 / 3}$ with the jet-medium coupling $\kappa_{\mathrm{sc}}^{q}=\kappa_{\mathrm{sc}}$ for quarks and $\kappa_{\mathrm{sc}}^{g}=\kappa_{\mathrm{sc}}\left(C_{A} / C_{F}\right)^{1 / 3}$ for gluons, including the respective Casimir operators $C_{A}$ and $C_{F}$.

This energy loss ansatz has been integrated into our existing model [5]. Please note that Ref. [8] uses natural units, $\hbar=c=1$. For a direct comparison, we quote our results below using a dimensionless coupling.

The main differences between the two energy-loss descriptions is the square-root dependence that leads to the formation of a Bragg peak with the explosive burst of energy close to the end of the jet's evolution. There have been discussions in literature [8, 10, 11] on the impact of the Bragg peak. In line with previous findings [10] we will show below that there is a difference between the Hybrid AdS energy-loss ansatz featuring a Bragg peak and the pQCD model without a Bragg peak, however, this difference is only marginal.

\section{Results and Discussion}

Fig. 1] shows the pion nuclear modification factor $\left(R_{A A}\right)$ for central (left panel) and mid-central (middle panel) collisions at RHIC (black) and LHC (red) as well as the high- $p_{T}$ elliptic flow $\left(v_{2}\right)$ for mid-central events (right). The measured data [1, 2, 3, 4] is compared to the pQCD-based energy loss of Eq. (1) with $(a=0, z=1, c=3 / 4)$. Jet-energy loss fluctuations $\left(\zeta_{0}\right)$ and the transverse expansion of the background flow $\left(v_{f}\right)$ are included, as well as a running jet-medium coupling that depends on the energy of the collision, $\kappa=\kappa\left(\sqrt{s_{N N}}\right)$.

Fig. 1 demonstrates that there is a surprising similarity between the results that cannot be expected a priori given the fact that the two background media are so different: While the hydrodynamic description of VISH2+1 [6] assumes an equilibrated system, the parton cascade BAMPS [7] also includes non-equilibrium effects in the bulk medium evolution.

In addition, the figure exhibits the so-called high$p_{T} v_{2}$-problem [5]: The high- $p_{T}$ elliptic flow below $p_{T} \sim 20 \mathrm{GeV}$ is about a factor of two below the measured data [1, 2, 3, 4]. This effect has been discussed in literature [5, 12, 13] and recently it has been suggested by CUJET3.0 [14] that a temperature and energydependent jet-medium coupling $\kappa=\kappa\left(E^{2}, T\right)$, which includes non-perturbative effects around the phase transition of $T_{c} \sim 160 \mathrm{MeV}$, can overcome this problem.

This jet-medium coupling was derived from the DGLV gluon number distribution [14] and is given by the analytic formul

$$
\begin{aligned}
\kappa\left(E^{2}, T\right)= & \alpha_{S}^{2}\left(E^{2}\right) \chi_{T}\left(f_{E}^{2}+f_{E}^{2} f_{M}^{2} \mu^{2} / E^{2}\right) \\
& -\left(1-\chi_{T}\right)\left(f_{M}^{2}+f_{E}^{2} f_{M}^{2} \mu^{2} / E^{2}\right) .
\end{aligned}
$$

It includes a running coupling $\alpha_{S}\left(E^{2}\right)=1 /(c+$ $\left.9 / 4 \pi \log \left(E^{2} / T_{c}^{2}\right)\right)$ with $c=1.05$, the Polyakov-loop suppression of the color-electric scattering [15] via $\chi_{T}=$ $c_{q} L+c_{g} L^{2}$ with pre-factors $c_{q}, c_{g}$ for quarks and gluons, and the Polyakov loop $L<1$ as parametrized from lattice QCD, as well as an enhancement of scattering due to the magnetic monopoles near the critical temperature 

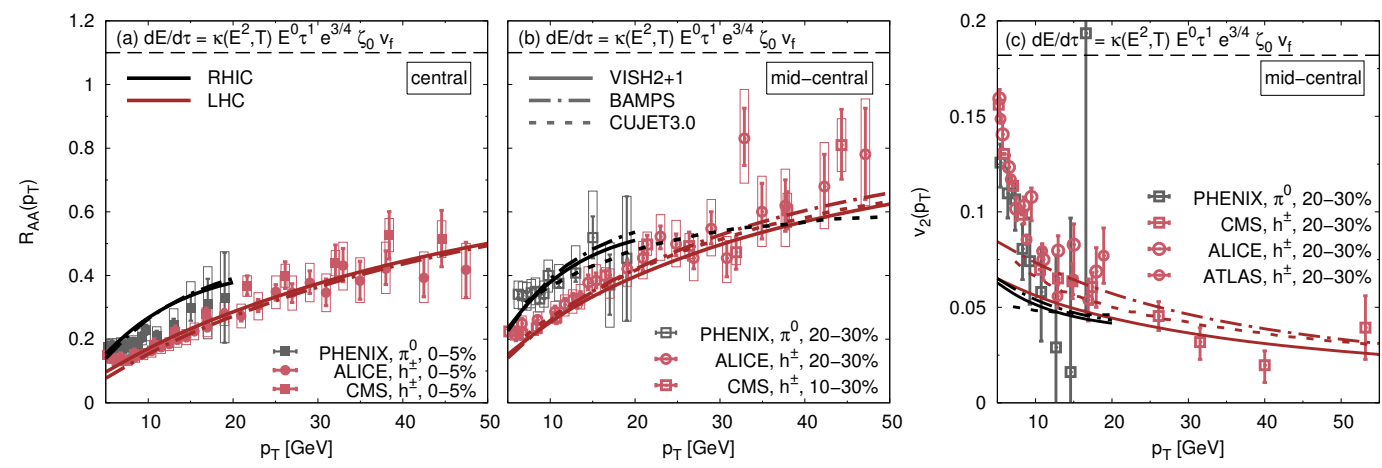

Figure 2: The pion nuclear modification factor for central (left panel) and mid-central (middle panel) collisions at RHIC (black) and LHC (red) as well as the high- $p_{T}$ pion elliptic flow for mid-central events (right panel). The measured data [1 2, 3. 4] is compared to a pQCD-based energy loss $d E / d \tau=\kappa\left(E^{2}, T\right) E^{0} \tau^{1} e^{3 / 4} \zeta_{0} v_{f}$, including jet-energy loss fluctuations $\left(\zeta_{0}\right)$ and transverse flow fields $\left(v_{f}\right)$, for a jet-medium coupling that depends on the energy of the jet, the temperature of the medium, and non-equilibrium effects around the phase transition $\left(\kappa=\kappa\left(E^{2}, T\right)\right)$ applying the hydrodynamic backgrounds of VISH2+1 [6] (solid lines) and the parton cascade BAMPS [7] (dashed-dotted lines). For comparison, we include the results of CUJET3.0 [14] (dotted lines).

$T_{c}$ also derived from lattice QCD [16]. This temperature and energy-dependent jet-medium coupling shows an effective running as it decreases with temperature.

We included the above jet-medium coupling $\kappa=$ $\kappa\left(E^{2}, T\right)$ in our jet-energy loss approach [5]. The result is shown in Fig. 2, again for the hydrodynamic background VISH2+1 (solid lines) and a medium determined via the parton cascade BAMPS (dashed lines). For comparison, we depict the results from CUJET3.0 [14].

As in Fig. 1, the ion nuclear modification factor is well described both at RHIC and LHC. The high$p_{T}$ elliptic flow, however, increases drastically below $p_{T} \sim 20 \mathrm{GeV}$ as compared to Fig. 1. especially for the BAMPS background which already includes nonequilibrium effects [7].

Finally, we compare results from the linear pQCD approach of Eq. (1) with the highly non-linear hybrid AdS holographic model of jet-energy loss, see Eq. (2). We compare the pion nuclear modification factor and an idealized LO Jet $R_{A A}$ given by

$$
\text { LO Jet } R_{A A}=\frac{R_{A A}^{g} d \sigma_{g}\left(p_{T}\right)+R_{A A}^{q} d \sigma_{q}\left(p_{T}\right)}{d \sigma_{g}\left(p_{T}\right)+d \sigma_{q}\left(p_{T}\right)} .
$$

Naturally, this LO Jet $R_{A A}$ represents a reconstructed jet with vanishing cone radius and is only a lower bound for the NLO Jet $R_{A A}$ with jet-cone radii $R>0$.

Fig. 3 shows this comparison at LHC (left) and RHIC (right) energies for two different jet-medium couplings that are treated as constants: A larger one (red) fitted to the pion $R_{A A}$ data (dashed-dotted lines) at RHIC and a lower one (blue) fitted the pion $R_{A A}$ data at LHC.

To guide the eye, we include the reconstructed Jet
$R_{A A}$ from CMS [17] with $R=0.3$ in Fig. 3. The solid blue lines for the LO Jet $R_{A A}$ in the left panels of Fig. 3 lie in the same ballpark as the experimental data. Fragmenting this result to pions (dashed-dotted lines) leads to an $R_{A A}$ that reproduces the measured pion nuclear modification factor at LHC. A straight extrapolation of this results to RHIC energies shows that the LO Jet $R_{A A}$ for the same jet medium couplings lie on top of the measured pion nuclear modification factor. However, fragmenting this result to pions leads to a $R_{A A}^{\pi}$ that is larger than the measured data at RHIC.

Larger jet-medium couplings (red lines), on the other hand, describe the pion nuclear modification factor at RHIC for the pQCD scenario and the LO Jet $R_{A A}$ at LHC is again close to the experimental data. The pion nuclear modification factor at LHC, however, only touches the lower bound of present error bars. In case of the Hybrid AdS energy loss the results always only touch the lower end of the experimental error bars.

Fig. 3 demonstrates that the results for the pQCD and the Hybrid AdS energy-loss including a Bragg peak are remarkably similar. Thus, unfortunately, neither the pion nor a LO Jet $R_{A A}$ are sensitive to the difference in the path-length between pQCD and AdS models.

\section{Conclusions}

We compared the measured data on the nuclear modification factor for pions and reconstructed jets as well as on the high- $p_{T}$ elliptic flow at RHIC and LHC energies to results obtained by a linear $\mathrm{pQCD}$ and a highly non-linear hybrid AdS holographic model of jet-energy loss. We found that the simultaneous description of the 

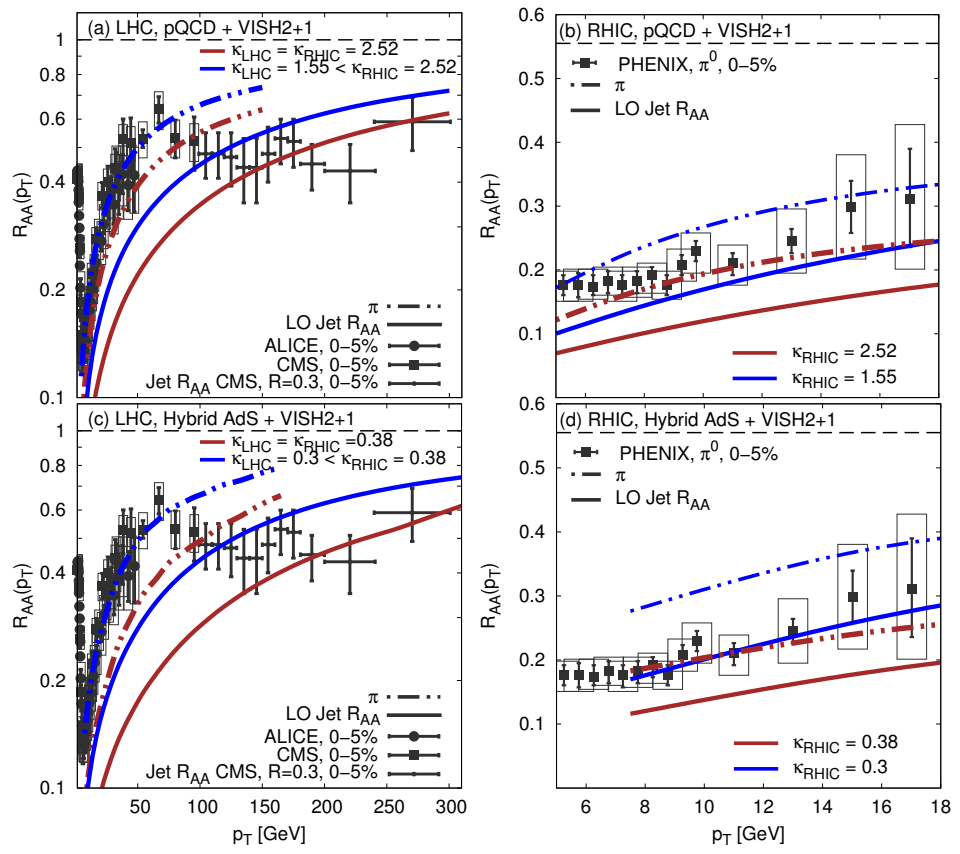

Figure 3: Comparison of the pion nuclear modification factor (dashed-dotted lines) and a LO Jet $R_{A A}$ (solid lines) calculated via a radiative pQCD-like energy-loss ansatz given by $d E / d \tau=\kappa E^{0} \tau^{1} e^{3 / 4} \zeta_{0} v_{f}$ (upper panels) with a constant jet-medium coupling and the hybrid strong/weak string energy-loss ansatz of Ref. [8] (lower panel) at LHC (left) and RHIC (right) energies for larger (red) and lower (blue) jet-medium couplings compared to the measured data [2, 3, 17].

$R_{A A}$ and $v_{2}$ requires a jet-medium coupling that depends on the energy of the jet, the temperature of the medium [5, 14], and non-equilibrium effects around the phase transition. We also contrasted a hydrodynamic background (VISH2+1) [6] with a medium obtained from the parton cascade BAMPS [7] and showed that the influence of the underlying bulk medium considered is suprisingly small. Unfortunately, neiter the pion nor the LO Jet $R_{A A}$ are sensitive to the difference in the pathlength between pQCD and AdS models.

\section{Acknowledgement}

This work was supported in part through the Bundesministerium für Bildung und Forschung, the Helmholtz International Centre for FAIR within the framework of the LOEWE program (Landesoffensive zur Entwicklung Wissenschaftlich-Ökonomischer Exzellenz) launched by the State of Hesse, the US-DOE Nuclear Science Grant No. DE-AC02-05CH11231 within the framework of the JET Topical Collaboration, and the US-DOE Nuclear Science Grant No. DE-FG0293ER40764. Numerical computations have been performed at the Center for Scientific Computing (CSC).

\section{References}

[1] A. Adare et al. [PHENIX Collaboration], Phys. Rev. C 87, 034911 (2013); Phys. Rev. Lett. 105, 142301 (2010).

[2] B. Abelev et al. [ALICE Collaboration], Phys. Lett. B 720, 52 (2013); Phys. Lett. B 719, 18 (2013).

[3] S. Chatrchyan et al. [CMS Collaboration], Eur. Phys. J. C 72, 1945 (2012); Phys. Rev. Lett. 109, 022301 (2012).

[4] G. Aad et al. [ATLAS Collaboration], Phys. Lett. B 707, 330 (2012).

[5] B. Betz and M. Gyulassy, arXiv:1503.07671 [hep-ph]; JHEP 1408, 090 (2014) [Erratum-ibid. 1410, 043 (2014)]; Phys. Rev. C 86, 024903 (2012)

[6] C. Shen, U. Heinz, P. Huovinen and H. Song, Phys. Rev. C 84, 044903 (2011); Phys. Rev. C 82, 054904 (2010).

[7] J. Uphoff et al., Phys. Rev. Lett. 114, no. 11, 112301 (2015); Z. Xu and C. Greiner, Phys. Rev. C 71, 064901 (2005).

[8] J. Casalderrey-Solana et al., JHEP 1410, 19 (2014).

[9] P. M. Chesler and K. Rajagopal, Phys. Rev. D 90, 025033 (2014).

[10] B. Betz et al., Phys. Rev. C 79, 034902 (2009).

[11] A. Ficnar, S. S. Gubser and M. Gyulassy, arXiv:1404.0935 [hepph]; Phys. Lett. B 738, 464 (2014); A. Ficnar and S. S. Gubser, Phys. Rev. D 89, 026002 (2014).

[12] J. Xu, A. Buzzatti and M. Gyulassy, JHEP 1408, 063 (2014)

[13] D. Molnar and D. Sun, arXiv:1305.1046 [nucl-th]; Nucl. Phys. A 910-911, 486 (2013).

[14] J. Xu, J. Liao and M. Gyulassy, Chin. Phys. Lett. 32, no. 9, $092501(2015)$

[15] Y. Hidaka and R. D. Pisarski, Phys. Rev. D 78, 071501 (2008).

[16] A. Nakamura, T. Saito and S. Sakai, Phys. Rev. D 69, 014506 (2004).

[17] CMS Collaboration [CMS Collaboration], CMS-PAS-HIN-12004. 